\title{
Biochemical network models simplified by balanced truncation
}

\author{
Wolfram Liebermeister ${ }^{1}$, Ulrike Baur ${ }^{2}$ and Edda Klipp ${ }^{1}$ \\ 1 Max Planck Institute for Molecular Genetics, Berlin, Germany \\ 2 Technical University Berlin, Institute of Mathematics, Berlin, Germany
}

\section{Keywords \\ balanced truncation; biochemical reaction system; complexity reduction; metabolic model; modularity}

\section{Correspondence}

W. Liebermeister, Max Planck Institute for Molecular Genetics, Ihnestraße 73,

14195 Berlin, Germany

Fax: +493080409322

Tel: +493080409318

E-mail: lieberme@molgen.mpg.de

Website: http://www.molgen.mpg.de/

ag_klipp/

(Received 24 December 2004, revised 10 May 2005, accepted 19 May 2005)

doi:10.1111/j.1742-4658.2005.04780.x
Modelling of biochemical systems usually focuses on certain pathways, while the concentrations of so-called external metabolites are considered fixed. This approximation ignores feedback loops mediated by the environment, that is, via external metabolites and reactions. To achieve a more realistic, dynamic description that is still numerically efficient, we propose a new methodology: the basic idea is to describe the environment by a linear effective model of adjustable dimensionality. In particular, we (a) split the entire model into a subsystem and its environment, (b) linearize the environment model around a steady state, and (c) reduce its dimensionality by balanced truncation, an established method for large-scale model reduction. The reduced variables describe the dynamic modes in the environment that dominate its interaction with the subsystem. We compute metabolic response coefficients that account for complexity-reduced dynamics of the environment. Our simulations show that a dynamic environment model can improve the simulation results considerably, even if the environment model has been drastically reduced and if its kinetic parameters are only approximately known. The speed-up in computation gained by model reduction may become vital for parameter estimation in large cell models.
Complexity reduction is an important issue in the mathematical modelling of cells. The use of small effective models can speed up numerical simulations considerably, and on top of this, focusing on the most important modes of dynamics can help us to understand the design of biological systems. In this article, we concentrate on small biochemical systems (e.g. a single metabolic pathway) that are embedded in a complex environment. For the sake of modelling, reactions in the environment are often ignored, while external metabolite concentrations are held at fixed values. To justify this, it is typically assumed that these metabolite concentrations are either very high or efficiently buffered, which is not always the case. If the buffering is incomplete, then the system will influence its environment and create perturbations that can act back on the system. If this feedback loop is neglected, then the model is possibly not suited to describe the data, and fitted model parameters may be wrong even if the fit looks satisfactory. Hence, we are looking for a more faithful representation of the environment that can provide realistic boundary conditions.

For the modelling of steady states, this has been accomplished by using phenomenological relations between different external metabolite concentrations [1]. For dynamic simulations, however, the problem becomes harder: the environment has to be modelled dynamically, which can increase the simulation time. As a remedy, we propose to replace it by a small linear model that is supposed to mimic the dynamic responses of the original environment. Reduction of linear models has been studied for a long time, and various methods have been proposed. We use balanced truncation [2], which is numerically demanding but yields a stable reduced system with a bounded approximation error (the Matlab code for balanced truncation can be found at http://www.tu-chemnitz.de/mathematik/ industrie_technik/software/software.php). Moreover, by tuning the dimensionality, one can choose a compromise between approximation accuracy and numerical efficiency. Balanced truncation has successfully been 
applied to linear control systems of high state-space dimensions ([3] and examples therein).

This article provides the reader with practical instructions for applying complexity reduction to biochemical models, and illustrates it with simple example models. An outline of balanced truncation is given in the methods section at the end of the article. We shall not touch upon the challenging question of how a detailed cell model can be established in the first place. Our goal is to make existing large models tractable and to speed up simulations, which can be vital for parameter estimation by maximum-likelihood or Bayesian methods (e.g. Monte Carlo Markov chain [4]). Besides this, balanced truncation highlights the dynamic modes of the environment that are most important for its interactions with the system under study — which may be interesting in itself.

\section{Model separation and reduction}

A common 'divide-and-conquer' approach to model reduction is to split the entire complex system into modules and to study them separately. It has been argued that biological systems have evolved to consist of weakly interacting modules (also termed 'pathways') because this may increase their robustness ([5] and references therein). There exist handy heuristics for defining modules in mathematical cell models, for instance cutting the network at 'hub' metabolites [6] and clustering the time series obtained from model simulations [7]. Rohwer et al. [8] defined monofunctional units for metabolism. Interactions among modules in steady state and the relationship between the local and global behaviour have been studied in modular response theory [9].

A second successful method of complexity reduction is to exploit the time scale separation of fast and slow processes [10,11]: by assuming quasi-steady states or quasi-equilibria, the number of independent variables can be reduced considerably, as exemplified by the analysis of the Wnt signalling pathway in [12]. Alternatively, fast global modes, as detected by analysing the Jacobian, can be eliminated 'on the flight' during simulations [13].

Here we examine a particular combination of modularization and complexity reduction: starting from a biochemical model, which comprises a subsystem and its environment, we aim to maintain the subsystem in its original form while replacing the environment by a linear model of lower dimensionality. We proceed as follows. First, the subsystem is split into an internal part and a boundary containing the communicating metabolites. Likewise, the environment is split into an exterior part and a boundary containing the communi- cating reactions. Subsystem and environment are only connected via the communicating metabolites and reactions, and the essence of our method is to provide the subsystem with approximate time courses of the communicating reactions, which in turn respond to the communicating metabolites. To simplify the relationship between them, we linearize the environment model around a stable steady state and replace it, using balanced truncation, by a small effective model. In the remainder of this section, we shall elucidate these points step by step.

\section{A metabolic system and its environment}

The modelling of metabolic networks has been described in detail by Heinrich and Schuster [10], and a convenient introduction to metabolic control analysis can be found in Hofmeyer [14]. Let us recall here just some basic definitions: a biochemical reaction system is described by the differential equation system

$$
\dot{\mathbf{s}}(t)=N \mathbf{v}(\mathbf{s}(t), \mathbf{p})
$$

where $\mathbf{s}$ is the vector of metabolite concentrations and $\mathbf{v}$ is the vector of reaction velocities. The vector $\mathbf{p}$ contains the kinetic parameters, and $N$ denotes the stoichiometric matrix, which contains in its $k^{\text {th }}$ column the stoichiometric coefficients for the $k^{\text {th }}$ reaction. An example can be found below. The derivatives $\left(\varepsilon_{\mathrm{s}}\right)_{i k}=$ $\partial v_{i} / \partial s_{k}$ are called the reaction elasticities. The parameter elasticities $\left(\varepsilon_{\mathrm{p}}\right)_{i m}=\partial v_{i} / \partial p_{m}$ are the derivatives of the reaction velocities with respect to the kinetic parameters.

The subsystem is defined by its metabolites, termed the subsystem metabolites. All other metabolites are called environment metabolites. Our first aim is to split a system into four regions, as shown in Fig. 1: the interior, the subsystem boundary (containing the communicating metabolites), the environment boundary (containing the communicating reactions), and the exterior. The interior and the exterior are connected to each other only via the boundaries. A metabolite and a reaction are called 'connected' if the metabolite is a substrate, product, or effector of the respective enzyme. We assign each metabolite and reaction either to the interior, to a boundary, or to the exterior by the following definitions: a reaction is called internal if it is only connected to subsystem metabolites, external if it is only connected to environment metabolites, and otherwise, it belongs to the environment boundary. A metabolite is called internal if it belongs to the subsystem and is only connected to internal reactions, external if it belongs to the environment, and otherwise, it belongs to the subsystem boundary. 


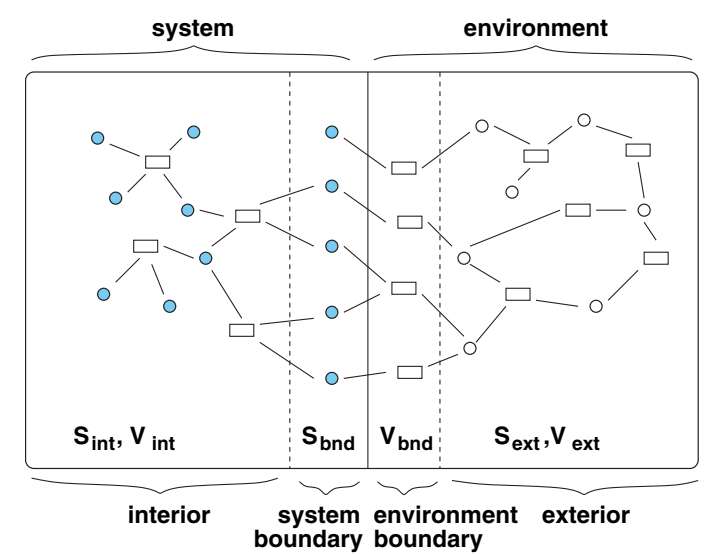

Fig. 1. Subdividing a biochemical network into subsystem and environment. Metabolites and reactions are shown as circles and boxes, respectively. The subsystem (left half) is defined by a set of metabolites (shaded circles). The entire system is split into four parts, the interior (left), the exterior (right), and the two boundaries (centre). The subsystem boundary consists of metabolites ( $\left.s_{\text {bnd }}\right)$, while the environment boundary consists of reactions $\left(v_{\text {bnd }}\right)$. The boundary metabolites connect the interior to the environment, and the boundary reactions connect the exterior to the subsystem.

Similar definitions apply if the subsystem is initially specified by its reactions. Internal, external and boundary quantities will be denoted by the subscripts 'int', 'ext', and 'bnd', respectively. The subscript 'tot' refers to the entire system.

After reordering the metabolites and reactions according to:

$$
\mathbf{s}_{\text {tot }}=\left(\begin{array}{c}
\mathbf{s}_{\text {int }} \\
\mathbf{s}_{\text {bnd }} \\
\mathbf{s}_{\text {ext }}
\end{array}\right), \quad \mathbf{v}_{\text {tot }}=\left(\begin{array}{c}
\mathbf{v}_{\text {int }} \\
\mathbf{v}_{\text {bnd }} \\
\mathbf{v}_{\text {ext }}
\end{array}\right),
$$

the above definitions imply that the stoichiometric matrix can be written as

$$
N_{\text {tot }}=\left(\begin{array}{lll}
N_{\text {int }}^{\text {int }} & 0 & 0 \\
N_{\text {int }}^{\text {bnd }} & N_{\text {bnd }}^{\text {bnd }} & 0 \\
0 & N_{\text {bnd }}^{\text {ext }} & N_{\text {ext }}^{\text {ext }}
\end{array}\right),
$$

and the vectors of reaction velocities for interior, boundary, and exterior read

$$
\begin{aligned}
\mathbf{v}_{\text {int }} & =\mathbf{v}_{\text {int }}\left(\mathbf{s}_{\text {int }}, \mathbf{s}_{\text {bnd }}, \mathbf{p}\right) \\
\mathbf{v}_{\text {bnd }} & =\mathbf{v}_{\text {bnd }}\left(\mathbf{s}_{\text {bnd }}, \mathbf{s}_{\text {ext }}, \mathbf{p}\right) \\
\mathbf{v}_{\text {ext }} & =\mathbf{v}_{\text {ext }}\left(\mathbf{s}_{\text {ext }}, \mathbf{p}\right) .
\end{aligned}
$$

The connections among metabolites and reactions in the four regions of the model are illustrated in Fig. 1. With Eqs (3) and (4), the system equations can be rewritten as

$$
\begin{aligned}
\dot{\mathbf{s}}_{\text {int }} & =N_{\text {int }}^{\text {int }} \mathbf{v}_{\text {int }}\left(\mathbf{s}_{\text {int }}, \mathbf{s}_{\text {bnd }}, \mathbf{p}\right) \\
\dot{\mathbf{s}}_{\text {bnd }} & =N_{\text {int }}^{\text {bnd }} \mathbf{v}_{\text {int }}\left(\mathbf{s}_{\text {int }}, \mathbf{s}_{\text {bnd }}, \mathbf{p}\right)+N_{\text {bnd }}^{\text {bnd }} \mathbf{v}_{\text {bnd }}\left(\mathbf{s}_{\text {bnd }}, \mathbf{s}_{\text {ext }}, \mathbf{p}\right) \\
\dot{\mathbf{s}}_{\text {ext }} & =N_{\text {ext }}^{\text {ext }} \mathbf{v}_{\text {ext }}\left(\mathbf{s}_{\text {ext }}, \mathbf{p}\right)+N_{\text {bnd }}^{\text {ext }} \mathbf{v}_{\text {bnd }}\left(\mathbf{s}_{\text {bnd }}, \mathbf{s}_{\text {ext }}, \mathbf{p}\right) .
\end{aligned}
$$

\section{Linearizing the environment model}

The next step is to linearize the reactions kinetics $\mathrm{v}_{\mathrm{bnd}}$ and $\mathrm{v}_{\mathrm{ext}}$ in the environment. To do so, we have to choose reference values $\overline{\mathbf{s}}_{\text {bnd }}, \overline{\mathbf{s}}_{\text {ext }}, \overline{\mathbf{v}}_{\text {bnd }}$, and $\overline{\mathbf{v}}_{\text {ext }}$, describing a steady state of the environment. The steady state requires that

$$
0=N_{\text {ext }}^{\text {ext }} \overline{\mathbf{v}}_{\text {ext }}+N_{\text {bnd }}^{\text {ext }} \overline{\mathbf{v}}_{\text {bnd }} .
$$

Valid reference values can be obtained as follows: we first choose some typical values for $\overline{\mathbf{s}}_{\text {bnd }}$ for the boundary metabolites and some reference values $\mathbf{p}^{0}$ for the kinetic parameters. Keeping these values fixed, we compute a steady state for the environment and accept the resulting steady-state concentrations and fluxes as the reference values $\overline{\mathbf{s}}_{\text {ext }}$ and $\overline{\mathbf{v}}_{\text {bnd }}$. If no stable steady state exists, then our approach cannot be implemented.

The reaction velocities in the environment are now linearized around the reference state, that is, replaced by the linear expressions

$$
\begin{aligned}
\mathbf{v}_{\text {bnd }}\left(\mathbf{s}_{\text {bnd }}, \mathbf{s}_{\text {ext }}, \mathbf{p}\right) & =\overline{\mathbf{v}}_{\text {bnd }}+\varepsilon_{\text {bnd }}^{\text {bnd }} \Delta \mathbf{s}_{\text {bnd }}+\varepsilon_{\text {ext }}^{\text {bnd }} \Delta \mathbf{s}_{\text {ext }}+\varepsilon_{\mathrm{p}}^{\text {bnd }} \Delta \mathbf{p} \\
\mathbf{v}_{\text {ext }}\left(\mathbf{s}_{\text {ext }}, \mathbf{p}\right) & =\overline{\mathbf{v}}_{\text {ext }}+\varepsilon_{\text {ext }}^{\text {ext }} \Delta \mathbf{s}_{\text {ext }}+\varepsilon_{\mathrm{p}}^{\text {ext }} \Delta \mathbf{p}
\end{aligned}
$$

where $\Delta \mathbf{s}_{\text {bnd }}:=\mathbf{s}_{\text {bnd }}-\overline{\mathbf{s}}_{\text {bnd }}$ and $\Delta \mathbf{s}_{\mathrm{ext}}:=\mathbf{s}_{\mathrm{ext}}-\overline{\mathbf{s}}_{\mathrm{ext}}$. The term $\Delta \mathbf{p}=\mathbf{p}-\mathbf{p}^{0}$ denotes a deviation from the reference parameter values. After setting $\Delta \mathbf{v}_{\text {bnd }}:=\mathbf{v}_{\text {bnd }}-$ $\overline{\mathbf{v}}_{\text {bnd }}$, the differential equations (Eqn 5) read

$$
\begin{aligned}
\dot{\mathbf{s}}_{\text {int }}= & N_{\text {int }}^{\text {int }} \mathbf{v}_{\text {int }}\left(\mathbf{s}_{\text {int }}, \mathbf{s}_{\text {bnd }}, \mathbf{p}\right) \\
\dot{\mathbf{s}}_{\text {bnd }}= & N_{\text {int }}^{\text {bnd }} \mathbf{v}_{\text {int }}\left(\mathbf{s}_{\text {int }}, \mathbf{s}_{\text {bnd }}, \mathbf{p}\right)+N_{\text {bnd }}^{\text {bnd }}\left(\overline{\mathbf{v}}_{\text {bnd }}+\Delta \mathbf{v}_{\text {bnd }}\right) \\
\Delta \dot{\mathbf{s}}_{\text {ext }}= & N_{\text {ext }}^{\text {ext }}\left(\overline{\mathbf{v}}_{\text {ext }}+\varepsilon_{\text {ext }}^{\text {ext }} \Delta \mathbf{s}_{\text {ext }}+\varepsilon_{\text {pt }}^{\text {ext }} \Delta \mathbf{p}\right) \\
& +N_{\text {bnd }}^{\text {ext }}\left(\overline{\mathbf{v}}_{\text {bnd }}+\varepsilon_{\text {bnd }}^{\text {bnd }} \Delta \mathbf{s}_{\text {bnd }}+\varepsilon_{\text {ext }}^{\text {bnd }} \Delta \mathbf{s}_{\text {ext }}+\varepsilon_{\text {p }}^{\text {bnd }} \Delta \mathbf{p}\right)
\end{aligned}
$$

with

$$
\Delta \mathbf{v}_{\text {bnd }}=\varepsilon_{\text {ext }}^{\text {bnd }} \Delta \mathbf{s}_{\text {ext }}+\varepsilon_{\text {bnd }}^{\text {bnd }} \Delta \mathbf{s}_{\text {bnd }}+\varepsilon_{\mathrm{p}}^{\text {bnd }} \Delta \mathbf{p} .
$$

With the stationarity condition (6), the third equation of (8) becomes:

$$
\begin{aligned}
\Delta \dot{\mathbf{s}}_{\mathrm{ext}}= & \left(N_{\mathrm{ext}}^{\mathrm{ext}} \varepsilon_{\text {ext }}^{\text {ext }}+N_{\text {bnd }}^{\text {ext }} \varepsilon_{\text {ext }}^{\text {bnd }}\right) \Delta \mathbf{s}_{\text {ext }} \\
& +N_{\text {bnd }}^{\text {ext }} \varepsilon_{\text {bnd }}^{\text {bnd }} \Delta \mathbf{s}_{\text {bnd }}+\left(N_{\text {ext }}^{\text {ext }} \varepsilon_{\mathrm{p}}^{\text {ext }}+N_{\text {bnd }}^{\text {ext }} \varepsilon_{\mathrm{p}}^{\text {bnd }}\right) \Delta \mathbf{p}
\end{aligned}
$$

For the sake of simplicity, let us assume that the parameters remain fixed $(\Delta \mathbf{p}=0)$. By setting

$$
\begin{aligned}
& \mathbf{x}=\Delta \mathbf{s}_{\text {ext }} \\
& \mathbf{u}=\Delta \mathbf{s}_{\text {bnd }} \\
& \mathbf{y}=\Delta \mathbf{v}_{\text {bnd }}
\end{aligned}
$$

and 


$$
\begin{aligned}
A & =N_{\mathrm{ext}}^{\mathrm{ext}} \varepsilon_{\mathrm{ext}}^{\text {ext }}+N_{\mathrm{bnd}}^{\mathrm{ext}} \varepsilon_{\mathrm{ext}}^{\mathrm{bnd}} \\
B & =N_{\mathrm{bnd}}^{\mathrm{ext}} \varepsilon_{\mathrm{bnd}}^{\mathrm{bnd}} \\
B_{\mathrm{p}} & =N_{\mathrm{bnd}}^{\mathrm{ext}} \varepsilon_{\mathrm{p}}^{\mathrm{bnd}}+N_{\mathrm{ext}}^{\mathrm{ext}} \varepsilon_{\mathrm{p}}^{\mathrm{ext}} \\
C & =\varepsilon_{\mathrm{ext}}^{\text {bnd }} \\
D & =\varepsilon_{\mathrm{bnd}}^{\text {bnd }} \\
D_{\mathrm{p}} & =\varepsilon_{\mathrm{p}}^{\text {bnd }}
\end{aligned}
$$

our equation system (10) can be written in a standard form for linear dynamical systems:

$$
\begin{gathered}
\dot{\mathbf{x}}(t)=A \mathbf{x}(t)+B \mathbf{u}(t), t>0, \mathbf{x}(0)=\mathbf{x}_{0} \\
\mathbf{y}(t)=C \mathbf{x}(t)+D \mathbf{u}(t), t \geq 0 .
\end{gathered}
$$

The first equation describes the dynamics of the external concentrations (x), depending on the changes of the boundary concentrations (u). The second equation expresses the boundary reaction velocities by the external and boundary concentrations. To account also for parameter changes $\Delta \mathbf{p}$, the above formulae need to be modified only slightly: we use the augmented vector $\mathbf{u}^{\prime}=\left(\begin{array}{c}\mathbf{u} \\ \Delta \mathbf{p}\end{array}\right)$ and the joint matrices $B^{\prime}=\left(\begin{array}{ll}B & B_{\mathrm{p}}\end{array}\right)$ and $D^{\prime}=\left(\begin{array}{ll}D & D_{\mathrm{p}}\end{array}\right)$.

For balanced truncation, the matrix $\mathrm{A}$, that is, the Jacobian of the environment model, must have full rank, which is not the case if the exterior concentrations $\mathrm{s}_{\text {ext }}$ obey conservation relations. In this case, we follow [15] and restrict the environment model to a set of independent environment metabolites with concentrations $\mathbf{s}_{\text {ind }}$. We define the reduced stoichiometric matrices $N_{\text {ext }}^{\text {ind }}$ and $N_{\text {bnd }}^{\text {ind }}$, and a link matrix $L$ such that $\dot{\mathbf{s}}_{\text {ext }}=L \dot{\mathbf{s}}_{\text {ind }}$. The expressions for $A, B$, and $C$ in (Eqn 12) are replaced by:

$$
\begin{aligned}
A & =N_{\text {ext }}^{\text {ind }} \varepsilon_{\text {ext }}^{\text {ext }} L+N_{\text {bnd }}^{\text {ind }} \varepsilon_{\text {ext }}^{\text {bnd }} L \\
B & =N_{\text {bnd }}^{\text {ind }} \varepsilon_{\text {bnd }}^{\text {bnd }} \\
B_{\mathrm{p}} & =N_{\text {bnd }}^{\text {ind }} \varepsilon_{\mathrm{p}}^{\text {bnd }}+N_{\text {ext }}^{\text {ind }} \varepsilon_{\mathrm{p}}^{\text {ext }} \\
C & =\varepsilon_{\text {ext }}^{\text {bnd }} L .
\end{aligned}
$$

This transformation to independent metabolites renders the matrix A nonsingular, except for pathologic cases where the steady-state of the environment is not stable. This happens if the elasticity matrix $\left(\varepsilon^{\mathrm{ext}} \mid \varepsilon^{\mathrm{bnd}}\right)$ does not have full column rank.

\section{Coupled system equations}

Now we can rewrite the entire system in a compact form: we drop the subscript for metabolites and reactions in the subsystem setting

$\mathbf{s}:=\left(\begin{array}{c}\mathbf{s}_{\text {int }} \\ \mathbf{s}_{\text {bnd }}\end{array}\right), \mathbf{v}=\mathbf{v}_{\text {int }}, N:=\left(\begin{array}{c}N_{\text {int }}^{\text {int }} \\ N_{\text {int }}^{\text {bnd }}\end{array}\right), N_{\text {bnd }}:=\left(\begin{array}{c}N_{\text {bnd }}^{\text {int }} \\ N_{\text {bnd }}^{\text {bnd }}\end{array}\right), \varepsilon:=\varepsilon_{\text {int }}^{\text {int }}$, and introduce a projection matrix $P$ such that $\mathbf{s}_{\mathrm{bnd}}=$ $P_{\text {Ss. }}$. Altogether, we obtain a coupled equation system for internal concentrations $\mathbf{s}$ and external concentrations $\mathbf{x}$ :

$$
\begin{aligned}
\mathbf{u}(t) & =P \mathbf{s}(t)-\overline{\mathbf{s}}_{\text {bnd }} \\
\dot{\mathbf{x}}(t) & =A \mathbf{x}(t)+B \mathbf{u}(t)+B_{\mathrm{p}} \Delta \mathbf{p}(t) \\
\mathbf{y}(t) & =C \mathbf{x}(t)+D \mathbf{u}(t)+D_{\mathrm{p}} \Delta \mathbf{p}(t) \\
\mathbf{v}_{\text {bnd }}(t) & =\overline{\mathbf{v}}_{\text {bnd }}+\mathbf{y}(t) \\
\dot{\mathbf{s}}(t) & =N \mathbf{v}(\mathbf{s}(t), \mathbf{p}(t))+N_{\text {bnd }} \mathbf{v}_{\text {bnd }}(t)
\end{aligned}
$$

with the initial condition $\mathbf{x}(0)=\mathbf{s}_{\text {ext }}(0)-\overline{\mathbf{s}}_{\text {ext }}$. The external metabolites $\mathbf{s}_{\text {ext }}$ are now hidden in the variables $\mathbf{x}$. Altogether, this equation system consists of: (a) a biochemical model describing the subsystem with external fluxes $\mathbf{v}_{\text {bnd }}$ (Eqn 15e); (b) a linear model of the standard form (Eqn 13), describing the environment (Eqns 15b and 15c) and (c) instructions on how to match both modules (Eqns 15a and 15d).

\section{Reducing the environment model}

After translating our model into the form (Eqn 15), we are ready to reduce the external concentrations to a smaller number of variables. The basic idea of model reduction as used here will be summarized in this paragraph: we consider a dynamic linear system of $n$ state variables $x_{i}$, which are controlled by $m$ input variables $u_{k}$ and can be observed via the $p$ output variables $y_{l}$. The time behaviour of $\mathbf{x}$ and $\mathbf{y}$ is described by a linear equation system of the form (Eqn 13) where the matrix $A$ is stable, that is, all its eigenvalues have negative real part. In this setting, $n$ is assumed to be quite large and the dimensions of the input and the output space are much smaller than $n(m, p \ll n)$. Without loss of generality, we have assumed that for $\mathbf{u}=0$, the system has a steady state at $\mathbf{x}=0$. For fixed initial conditions, any time course $\mathbf{u}(\cdot)$ of the controlling variables leads to a time course $\mathbf{y}(\cdot)$ of the observables.

In model reduction, we aim at replacing the system (Eqn 13) by a lower-dimensional system of order $r$ $(r \ll n)$ that yields a good approximation of the inputoutput relationship. First of all, the input-output relationship can be exactly represented by a system with transformed variables $\tilde{\mathbf{x}}$. If $T$ is an invertible $n \times n$ matrix, we can apply the transformation:

$$
\begin{aligned}
\mathbf{x} \rightarrow \tilde{\mathbf{x}} & =T \mathbf{x} \\
A \rightarrow \tilde{A} & =T A T^{-1} \\
B \rightarrow \tilde{B} & =T B \\
C \rightarrow \tilde{C} & =C T^{-1}
\end{aligned}
$$

without changing the input-output relation between $\mathbf{u}(\cdot)$ and $\mathbf{y}(\cdot)$. Of course, the initial value $\mathbf{x}_{0}$ must also 
be transformed. For a chosen dimensionality $r$, we can now split $T=\left(\begin{array}{l}T_{1} \\ T_{2}\end{array}\right), T^{-1}=\left(S_{1} S_{2}\right)$ with an $r \times n$ matrix $T_{1}$ and an $n \times r$ matrix $S_{1}$. The transformation

$$
\begin{aligned}
& \mathbf{x} \rightarrow \tilde{\mathbf{x}}=T_{1} \mathbf{x} \\
& A \rightarrow \tilde{A}=T_{1} A S_{1} \\
& B \rightarrow \tilde{B}=T_{1} B \\
& C \rightarrow \tilde{C}=C S_{1}
\end{aligned}
$$

yields a reduced model of dimension $r$

$$
\begin{array}{ll}
\dot{\tilde{\mathbf{x}}}(t)=\tilde{A} \tilde{\mathbf{x}}(t)+\tilde{B} \mathbf{u}(t), & t>0, \tilde{\mathbf{x}}(0)=\tilde{x}_{0} \\
\tilde{\mathbf{y}}(t)=\tilde{C} \tilde{\mathbf{x}}(t), \tilde{\mathbf{y}}(t)=\tilde{C} \tilde{\mathbf{x}}(t)+\tilde{D} \tilde{\mathbf{u}}(t), & t \geq 0
\end{array}
$$

that approximates the input-output relation. We use balanced truncation [2] to find reduced representations $\tilde{A}, \tilde{B}, \tilde{C}, \tilde{D}$ that yield a good approximation of the full system. The basic idea behind balanced truncation is outlined in the methods section.

\section{Response coefficients}

Metabolic response coefficients can be computed for a reduced system of the form Eqn 15. We assume that for some reference choice $\mathbf{p}=\mathbf{p}^{0}$ of the parameter vector, the equation system has a stable steady state at $\left(\begin{array}{l}\mathbf{s}_{\mathrm{ss}} \\ \mathbf{x}_{\mathrm{ss}}\end{array}\right)$ with stationary fluxes $\mathbf{v}_{\mathrm{ss}}=\mathbf{v}\left(\mathbf{s}_{\mathbf{s s}}, \mathbf{p}\right)$. The matrices of metabolic response coefficients are defined as the derivatives $R^{\mathrm{S}}=\partial \mathbf{s}_{\mathrm{ss}} / \partial \mathbf{p}, \quad R^{\mathrm{J}}=\partial \mathbf{v}_{\mathrm{ss}} / \partial \mathbf{p}, \quad R^{\mathrm{x}}=\partial \mathbf{x}_{\mathrm{ss}} / \partial \mathbf{p}$ of the steady-state quantities $\mathbf{s}_{\mathrm{ss}}, \mathbf{x}_{\mathrm{ss}}$, and $\mathbf{v}_{\mathrm{ss}}$ with respect to the parameters $\mathbf{p}$. The matrix of partial derivatives is defined by $(\partial \mathbf{y} / \partial \mathbf{x})_{i k}:=\partial y_{i} / \partial x_{k}$. The response coefficients read:

$$
\begin{gathered}
R^{\mathrm{S}=}-\left[N \varepsilon+N_{\text {bnd }}\left(D-C A^{-1} B\right) P\right]^{-1} \\
\times\left[N \varepsilon_{\mathrm{p}}+N_{\text {bnd }}\left(D \mathbf{p}-C A^{-1} B_{\mathrm{p}}\right)\right] \\
R^{\mathrm{X}}=-A^{-1}\left(B P R^{\mathrm{S}}+B_{\mathrm{p}}\right) \\
R^{\mathrm{J}}=\varepsilon R^{\mathrm{S}}+\varepsilon_{\mathrm{p}}
\end{gathered}
$$

if the matrix inverse in Eqn 18 exists. (The derivation can be found in the Appendix). Traditionally, $R^{\mathrm{S}}$ and $R^{\mathrm{J}}$ have been computed for systems with a fixed environment [10]. Equation 18 differs from the known formula

$$
R^{\mathrm{S}}=-(N \varepsilon)^{-1} N \varepsilon_{\mathrm{p}}
$$

by the additional term $N_{\text {bnd }}\left(D-C A^{-1} B\right) P$ in Eqn 18 , which describes a feedback via the environment, and by the term $N_{\text {bnd }}\left(D_{\mathrm{p}}-C A^{-1} B_{\mathrm{p}}\right)$ describing the parameters' influence on the environment. If the connections between subsystem and environment are neglected, for instance, if $N_{\text {bnd }}$ vanishes, then the standard formula (Eqn 21) is reobtained.

\section{Examples}

\section{Small reaction chain}

To illustrate the whole process of model splitting, linearization, and reduction, we consider a small chain of four metabolites S1, S2, S3, and S4:

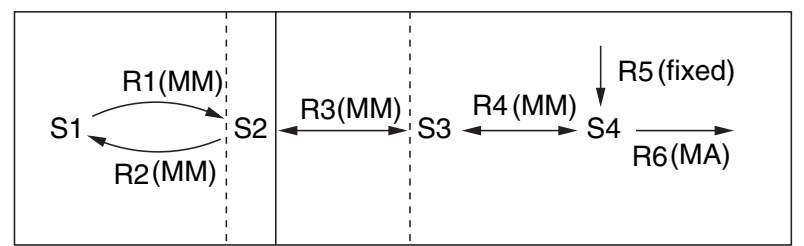

The reactions $\mathrm{R} 1$ and $\mathrm{R} 2$, which are catalysed by different enzymes, follow irreversible Michaelis-Menten kinetics (MM). Reactions R3 and R4 follow reversible Michaelis-Menten kinetics (MM), reaction R5 is a fixed inflow, and reaction R6 is irreversible with massaction kinetics (MA).

The time courses of the metabolite concentrations obey the differential Eqn 1 with the stoichiometric matrix:

$$
N=\left(\begin{array}{rrrrrr}
-1 & 1 & 0 & 0 & 0 & 0 \\
1 & -1 & -1 & 0 & 0 & 0 \\
0 & 0 & 1 & -1 & 0 & 0 \\
0 & 0 & 0 & 1 & 1 & 1
\end{array}\right)
$$

and the reaction velocities

$$
\begin{aligned}
v_{1}= & V_{1} s_{1} /\left(K_{1}^{M}+s_{1}\right) \\
v_{2}= & V_{2} s_{2} /\left(K_{2}^{M}+s_{2}\right) \\
v_{3}= & \frac{\frac{V_{3}^{+}}{K_{3}^{+}} s_{2}-\frac{V_{3}^{-}}{K_{3}^{-}} s_{3}}{1+\frac{s_{2}}{K_{3}^{+}}+\frac{s_{3}}{K_{3}^{-}}} \\
v_{4}= & \frac{\frac{V_{4}^{+}}{K_{4}^{+}} s_{3}-\frac{V_{4}^{-}}{K_{4}^{-}} s_{4}}{1+\frac{s_{3}}{K_{4}^{+}}+\frac{s_{4}}{K_{4}^{-}}} \\
v_{5}= & V_{5} \\
v_{6}= & k_{6} s_{4}
\end{aligned}
$$

For simplicity, all kinetic parameters were set to 1 $\left(V_{1}, K_{1}^{M}, V_{2}, K_{2}^{M}, V_{3}^{+}, K_{3}^{+}, V_{3}^{-}, K_{3}^{-}, V_{4}^{+}, K_{4}^{+}, V_{4}^{-}, K_{4}^{-}, V_{5}, k_{6}\right)$. The vectors of stationary concentrations and fluxes read $S=(1,1,1,1)^{\mathrm{T}}$ and $J=(1 / 2,1 / 2,0,0,1,1)^{\mathrm{T}}$, respectively. For model reduction, we assume that metabolites $\mathrm{S} 1$ and $\mathrm{S} 2$ and reactions $\mathrm{R} 1$ and $\mathrm{R} 2$ form the subsystem, with metabolite $\mathrm{S} 2$ as the communicating metabolite, while the remaining metabolites and reactions form the environment. In the above model scheme, this is indicated by boxes just like in Fig. 1. The matrices in the equation system (Eqn 15) then read: 


$$
\begin{aligned}
P & =(0,1) \\
N & =\left(\begin{array}{rr}
-1 & 1 \\
1 & -1
\end{array}\right) \\
N_{\text {bnd }} & =(0,-1)^{\mathrm{T}} \\
A & =1 / 3\left(\begin{array}{rr}
-2 & 1 \\
1 & -4
\end{array}\right) \\
B & =1 / 3(1,0)^{\mathrm{T}} \\
C & =1 / 3(-1,0) \\
D & =1 / 3
\end{aligned}
$$

Figure 2 shows simulation results for the system and different reduced versions of it. The calculation, and all the following ones, were done in MATLAB. Initially, all variables were set to half of the full system's respective steady-state concentrations. The figure shows time courses from the full model $(O)$, from the isolated subsystem (--), and from a larger isolated subsystem containing metabolites $\mathrm{S} 1, \mathrm{~S} 2$, and $\mathrm{S} 3$ (*). $^{*}$. Further, we consider the model with a linearized environment without dimension reduction (dotted), as well as reduced models with dimensions $0(\cdot-\cdot)$ and $1(-)$. The simulations show that the linearized model yields a good approximation of the full model, after being reduced to only one dimension. The isolated subsystem
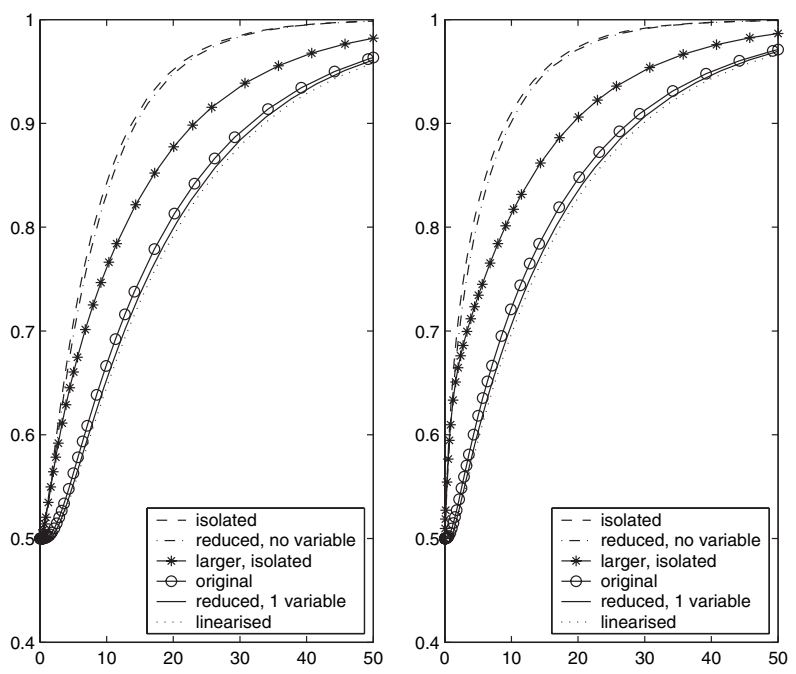

Fig. 2. Model reduction of a small chain of reactions (see text). The reduced model yields an excellent approximation, while imposing fixed external concentrations compromises the simulation results considerably. Left, time courses of variable S1. The lines represent different models: the isolated subsystem with fixed environment $(---)$, the reduced model with no environment variables $(\cdot-\cdot)$, the isolated subsystem containing metabolites S1, S2, and S3 $\left({ }^{*}\right)$, the full model (solid line with circles), the reduced model with dimension $1(-)$, and the model with a linearized environment ( . . .). Right, the same, for variable 2 . Time and concentrations are measured in arbitrary units.
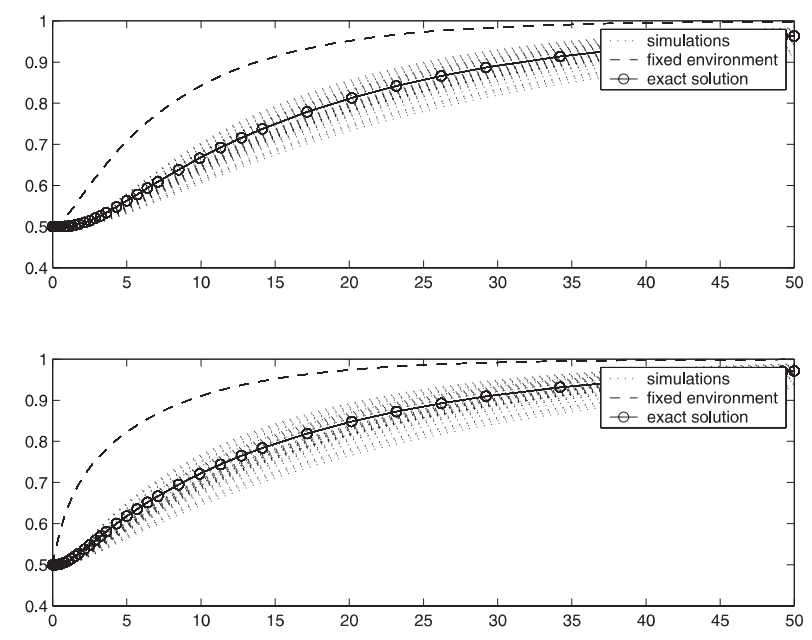

Fig. 3. Modelling an environment with parameter uncertainty. The diagrams show simulation results from the same metabolic model as in Fig. 2. Top, the solid line with circles shows time courses of the variable S1. Even with noisy parameters (see text), all reduced models yield a better approximation (50 simulation runs, shown by dots) of the true dynamics than a model with fixed external concentrations (dashed line). Bottom, the same, for variable S2.

and the reduced system with no effective variables yield much steeper time curves, while the larger subsystem, treated in isolation, yields intermediate results.

It is an interesting question whether a model of the environment should be taken into account even if it is not fully reliable. To elucidate this for the present example, we studied the effect of parameter uncertainties in the environment. Figure 3 compares the isolated model (dashed) to different simulations of the reduced model (1 dimension) with random choices of the parameters. To obtain a fair comparison, we ensured that both kinds of models yield the same steady state. Hence, we chose the random parameters as follows: three random numbers $z_{1}, z_{2}, z_{3}$ were chosen independently in the range between 0.5 and 2, with uniform logarithmic values. Then all parameters of reaction R3 were scaled (multiplied) by $z_{1}$, all parameters of reaction $\mathrm{R} 4$ were scaled by $z_{2}$, and the parameters of reactions R5 and R6 were scaled by $z_{3}$. Figure 3 shows that, despite the noisy parameters, all reduced models (50 simulations) yield better approximations of the true dynamics $(-)$ than the model with fixed external concentrations (-- -).

\section{Interpreting the variables in terms of external concentrations}

Each of the reduced variables obtained by balanced truncation represents a certain linear combination of the original external variables. To illustrate the meaning of the reduced variables, we consider a subdivision of the 

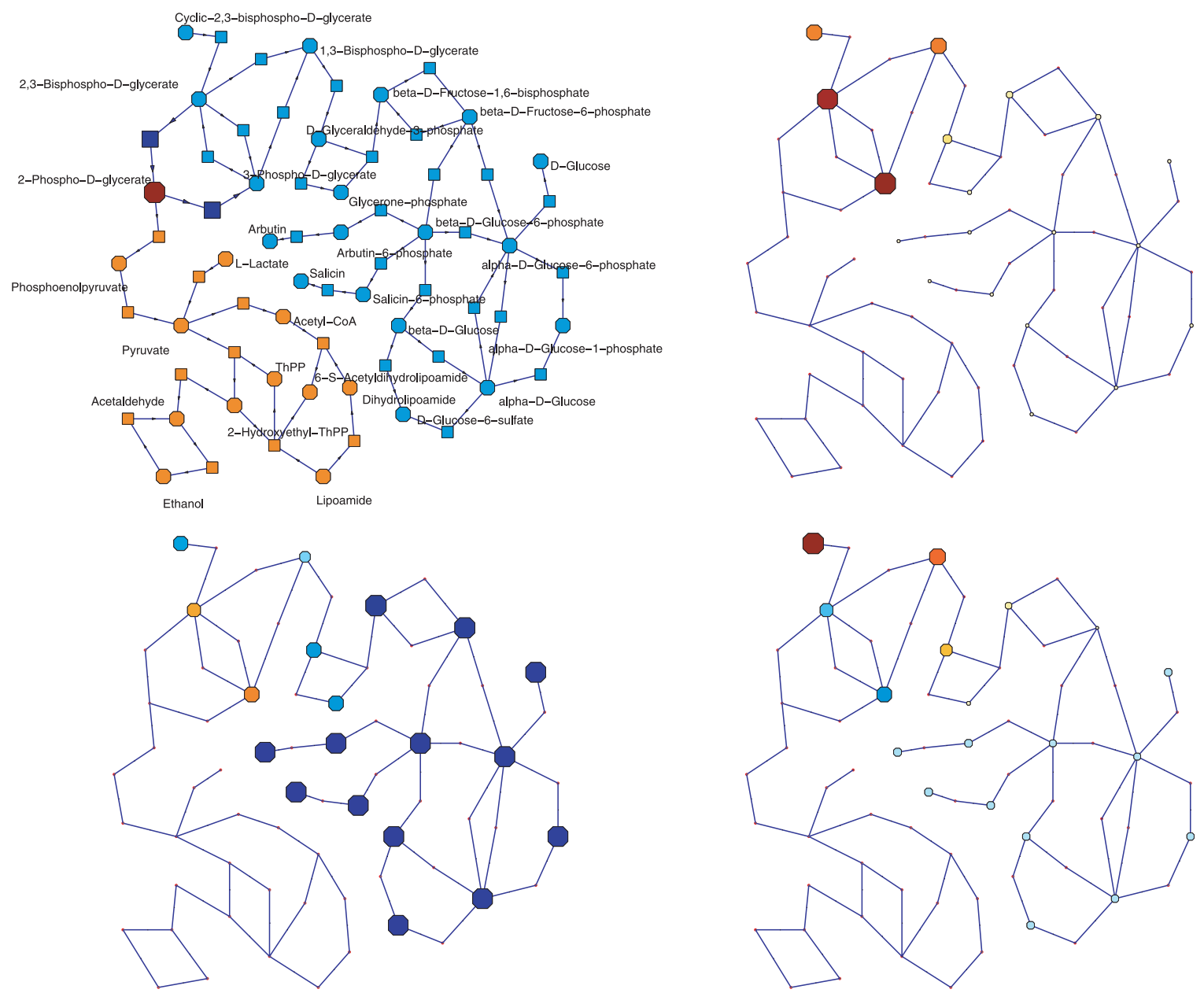

Fig. 4. Reduced variables in a biochemical network. Top left, glycolysis network from KEGG [16]. In this example, reactions are described by mass-action kinetics with arbitrary parameters (all values equal 1). The model consists of a subsystem under study (phosphoenolpyruvate and downstream) and an environment (the rest). The entire network is split into regions (compare Fig. 1) indicated by colours: interior (orange), the subsystem boundary (brown), the environment boundary (dark blue), and the exterior (light blue). Right, transformation weights for the first three external variables (1, top right; 2 , bottom left; 3 , bottom right). Positive and negative values are shown by the reddish and bluish colours, respectively, while the circle areas denote the absolute values (arbitrary scaling). With the first and third variable, the metabolites near the boundary carry the highest weights. The sign of the second variable changes between metabolites close to and far from the border.

glycolysis pathway, defined according to the KEGG database [16] (Fig. 4, top left). The aim of this analysis is not to model glycolysis with realistic kinetics, but to illustrate the transformation to reduced variables for a realistic metabolic network topology. A part of the network (2-phospho-D-glycerate and below) was arbitrarily chosen as the subsystem, while all upstream reactions form the environment. For simplicity, we assumed reversible mass-action kinetics with $k_{+}=\mathrm{k}_{-}=1$ for all reactions. After computing the steady state, we transformed the environment to balanced coordinates. The transformation matrix $S_{1}$ in Eqn 16 represents an approximate mapping from the transformed variables to the original variables, that is, the external metabolites. Figure 4 shows the transformation weights (columns of $\left.S_{1}\right)$ for the leading three variables $\left(x_{1}\right.$, top right; $x_{2}$, bottom left; $x_{3}$, bottom right). It turns out that the first and third variable represent mainly metabolites near the boundary, while the second variable represents a mode in which 2,3-bisphospho-D-glycerate and 3phospho-D-glycerate at the boundary are increased, while all other metabolites are decreased. This localization at the boundary may be a general feature of the dynamical modes that couple biochemical subsystems.

\section{Discussion}

Disintegration of metabolic models into subsystems has been pioneered by modular response theory $[9,17]$, which studies how the steady state of modular systems 
responds to changes of the model parameters. The analysis consists of two steps: first, the individual subsystems are described by effective, linear input-output relationships. Second, the modules are coupled based on their input-output relationships while all variables internal to the modules can remain hidden. Also in our dynamic approach, subsystems interact via a few communicating metabolites while the remaining variables are hidden inside the modules. A large model is split into subsystems, and linearization and complexity-reduction are applied to those - possibly large parts that are not in the focus of interest. In contrast to modular response theory, we retain a dynamic description of the environment, which represents the most important modes of dynamics around a steady state. We also characterized the steady-state behaviour of the reduced system by metabolic response coefficients.

Selective model reduction combines advantages of large-scale modelling with the modelling of isolated, well-understood systems. The compromise between numerical effort and approximation error can be tuned by choosing the dimensionality of the environment model. In this article, we considered a splitting into only two parts: one module that is maintained and another module to be reduced. Of course, the method readily applies to larger numbers of modules. The main assumptions are that: (a) the environment model exhibits a stable steady state for the given kinetic parameters; and (b) that the perturbations exerted by the subsystem are small.

Aiming at model reduction, we chose balanced truncation for a number of reasons: it allows for controlling the output error, that is, the difference of outputs between the original and the reduced system for the same input. Model reduction techniques can be applied to large systems: so far, we considered an algorithm implemented in MATLAB. With the corresponding SLICOT routines [18], systems of about 2000 variables can be reduced on a desktop computer with a memory capacity of $1 \mathrm{~GB}$. The extension PSLICOT for parallel computing [19] can deal with dimensions of several tens of thousands on small PC clusters. To exploit some special structure of the underlying system, for instance, sparsity of the system matrix, specialized methods like ADI-based iterative methods [20] or methods based on hierarchical matrix arithmetic [21] can be applied.

Model reduction does not preserve conservation relations that couple metabolites from both subsystem and environment. This is quite natural because the environment variables are no longer individually described, so these conservation relations actually lose their meaning. However, a loss of the conservation relations can have a visible effect on individual subsys- tem metabolites: they may exceed maximal concentrations set by the initial conditions. Let us illustrate this by a hypothetical example: consider a network containing a reaction $\mathrm{A}+\mathrm{ADP} \rightarrow \mathrm{B}+\mathrm{AMP}$ and an energy-supplying reaction ATP $\leftrightarrow$ ADP (where inorganic phosphate is not explicitly considered). The remaining reactions are not related to ATP, ADP, or AMP. If all metabolites are modelled explicitly, the concentrations $c_{\mathrm{ATP}}, c_{\mathrm{ADP}}$, and $c_{\mathrm{AMP}}$ form a conservation relationship - their sum remains constant. Let us assume that the energy source ATP starts with a high concentration and is converted into ADP and AMP. As ATP goes down, the supplying reaction will become slower, and $c_{\mathrm{ADP}}+c_{\mathrm{AMP}}$ stops rising before it reaches the upper limit set by $c_{\mathrm{AMP}}(0)+$ $c_{\mathrm{AMP}}(0)+c_{\mathrm{AMP}}(0)$. What happens if ATP is treated as an external metabolite? Again, the levels of ADP and AMP start rising, but ATP does not decrease, and at some time point, $c_{\mathrm{ADP}}(t)+c_{\mathrm{AMP}}(t)$ may exceed their upper limit. The fixed concentration of ATP leads to a bad approximation of the supplying reaction, which keeps on delivering ATP after the limit is reached. With a reduced environment model, we can generally expect a better approximation of the communicating flux velocities: the conservation relation will still be violated, but to a smaller extent-and again, the approximation error can be controlled by the choosing the dimensionality. Nevertheless, if a certain conservation relation has to be exactly fulfilled, then all participating metabolites should be included into the subsystem.

What is the meaning of the reduced variables in biochemical systems? Practically, they represent correction terms beyond the assumption of fixed external metabolites. Unlike the eigenmodes of the Jacobian [13], they are chosen such as to optimally mimic the behaviour of the environment, as seen by the subsystem. Interestingly, the first reduced variables in the glycolysis network accounted for differences between the boundary layer and the more distant parts. This may be explained by the fact that perturbations are damped and do not penetrate deeply into the environment, and that this aspect of the dynamics is then emphasized by balanced truncation. Consequently, we can expect that distant parts of the environment will influence the subsystem's dynamics only weakly, and that their exact modelling is probably of minor importance. In reverse, this might also justify the very fact that we started with a certain finite-sized environment, and not an even larger model.

One may argue that currently, the crucial issue in cell modelling is not the numerical effort of simulations, but the lack of kinetic data that are necessary to build the models. So why do we need model reduction at all? First, it should be noted that the speed-up in 
simulations can be quite considerable: once a reduced model with much smaller dimension than the original system has been established, further computations require much less storage and $\mathrm{CPU}$ time. This can become crucial in parameter fitting with maximum likelihood or MCMC methods, which require a large number of iterated simulation runs. For parameter fitting, several scenarios are conceivable: (a) an environment with fixed parameters is reduced to speed up the estimation of the subsystem's parameters; (b) some of the environment parameters remain unspecified during model reduction, in order to estimate them later along with the subsystem parameters; (c) The matrices A, B, $\mathrm{C}$, and $\mathrm{D}$ are regarded as effective parameters without referring to a specific environment model and are fitted together with the subsystem parameters. Second, our simulations show that accounting for the environment can improve the modelling results considerably, even if the kinetic parameters are not exactly known. Thus even in a stage where no reliable model parameters for the environment are available, a model-reduction approach may outperform the modelling of isolated subsystems with fixed external concentrations.

\section{Methods}

\section{Balanced truncation}

Closely connected with the stable, continuous-time system (Eqn 13) are the two matrices $\mathbf{P}$ and $\mathbf{Q}$, the infinite reachability Gramian and the infinite observability Grampian:

$$
\mathcal{P}:=\int_{0}^{\infty} \mathrm{e}^{A t} B B^{T} \mathrm{e}^{\mathrm{A}^{T_{t}}} \mathrm{~d} t, \quad \mathcal{Q}:=\int_{0}^{\infty} \mathrm{e}^{\mathrm{A}^{T} t} \mathrm{C}^{T} \mathrm{Ce}^{\mathrm{At}} \mathrm{d} t
$$

The Gramians can be interpreted in terms of energies: the minimal control energy $\mathcal{E}_{\mathrm{c}}$ for the transfer from the zero state to a state $\bar{x}$ over infinite horizon is

$$
\mathcal{E}_{\mathrm{c}}^{2}:=\inf _{u \in \mathcal{L}_{2}} \int_{0}^{\infty} u(t)^{T} u(t) \mathrm{d} t=\tilde{x}^{T} \mathcal{P}^{-1} \tilde{x}
$$

whereas the largest observation energy $\mathcal{E}_{\mathrm{c}}$ produced by observing the output of the system with initial state $x o$ over infinite horizon is

$$
\mathcal{E}_{o}^{2}:=\sup _{y \in \mathcal{L}_{2}} \int_{0}^{\infty} y(t)^{T} y(t) \mathrm{d} t=x_{0}^{T} \mathcal{Q} x_{0} .
$$

Model reduction by balanced truncation [2] is based on a special transformation into so-called balanced coordinates. The basic concept of balancing is finding a basis in which the two Gramians are equal and diagonal

$$
\mathcal{P}=\mathcal{Q}=\operatorname{diag}\left(\sigma_{1}, \ldots, \sigma_{n}\right),
$$

with ordered diagonal entries, the Hankel singular values of the system. In these new coordinates, states that are diffi- cult to control are also difficult to observe and vice versa. Model reduction by balanced truncation removes these state components; they are the states which are least involved in the energy transfer

$$
E:=\sup _{u \in \mathcal{L}_{2}} \frac{\int_{0}^{\infty} y(t)^{T} y(t)}{\int_{-\infty}^{0} u(t)^{T} u(t) \mathrm{d} t}=\frac{x_{0}^{T} \mathcal{Q} x_{0}}{x_{0}^{T} \mathcal{P}^{-1} x_{0}}
$$

from past inputs $\mathrm{u}$ to future outputs $\mathrm{y}$. The norm of the error is bounded by twice the sum of the neglected singular values

$$
\sup _{u \in \mathcal{L}_{2}} \frac{\|y-\tilde{y}\|_{2}}{\|u\|_{2}} \leq 2 \sum_{k=r+1}^{n} \sigma_{k}
$$

The balancing transformation $T$, in particular the parts $T_{1}$ and $S_{1}$ of the transformation mentioned in 'Reducing the environment model', are computed via the Cholesky factors of the two Gramians $\mathcal{P}$ and $\mathcal{Q}$ :

$$
\mathcal{P}=S^{T} S, \mathcal{Q}=R^{T} R
$$

We obtain the two transformation matrices after a singular value decomposition of $S R^{T}$ :

$$
S R^{T}=\left(U_{1} U_{2}\right)\left(\begin{array}{cc}
\Sigma_{1} & 0 \\
0 & \Sigma_{2}
\end{array}\right)\left(\begin{array}{l}
V_{1}^{T} \\
V_{2}^{T}
\end{array}\right), \Sigma_{1}=\operatorname{diag}\left(\sigma_{1}^{2}, \ldots, \sigma_{r}^{2}\right)
$$

By

$$
T_{1}=\Sigma_{1}^{-\frac{1}{2}} V_{1}^{T} R \quad \text { and } \quad S_{1}=S^{T} U_{1} \Sigma_{1}^{-\frac{1}{2}} .
$$

Model reduction by balanced truncation has some desirable properties: the reduced system (Eqn 17) remains stable and has a low approximation error with an a priori known upper bound. Therefore, the size of the reduced system can be chosen adaptively depending on the permitted error size. If we are interested in preserving the passivity of the system, that is, to obtain a reduced system which cannot produce energy internally, we have to apply another model reduction routine called positive real balancing (see [22] and references therein). Another class of model reduction methods are the Krylov-based methods. These methods do not preserve stability, have no given error bound, but have good numerical properties, (see [23]). For a broad collection of survey papers on model reduction, see [20], where also a couple of benchmark examples are presented.

\section{Acknowledgements}

The authors would like to thank W. Huisinga and P. Benner for support and insightful discussions. This work was supported by the Federal Ministry of Education and Research, by the DFG Research Center MATHEON 'Mathematics for key technologies' in Berlin, and by the European commission, grant no. 503269 . 


\section{References}

1 Petersen S, v Lieres E, de Graaf AA, Sahm H, \& Wiechert W (2004) Metabolic engineering in the post genomic era. In Multi-Scale Approach for the Predictive Modeling of Metabolic Regulation (Kholodenko BN \& Westerhoff HV eds), Horizon Bioscience.

2 Moore BC (1981) Principal component analysis in linear systems: controllability, observability and model reduction. IEEETrans AC AC-26, 17-32.

3 Antoulas AC \& Sorensen DC (2001) Approximation of large-scale dynamical systems: an overview. Int $J$ Appl

Math Comp Sci 11, 1093-1121.

4 Gelman A, Carlin JB, Stern HS \& Rubin DB (1997) Bayesian Data Analysis. Chapman \& Hall.

5 Stelling J, Sauer U, Szallasi Z, Doyle FJ \& Doyle J (2004) Robustness of cellular functions. Cell 118, 675-685.

6 Schuster S, Pfeiffer T, Moldenhauer F, Koch I \& Dandekar T (2002) Exploring the pathway structure of metabolism decomposition into subnetworks and application to Mycoplasma pneumoniae. Bioinformatics 18 (2), 351-361.

7 Ederer M, Sauter T, Bullinger E, Gilles E \& Allgöwer F (2003) An approach for dividing models of biological reaction networks into functional units. Simulation 79 (12), 703-716.

8 Rohwer JM, Schuster S, \& Westerhoff HV (1996) How to recognize monofunctional units in a metabolic system. J Theor Biol 179 (3), 213-228.

9 Bruggeman F, Westerhoff HV, Hoek JB, \& Kholodenko B (2002) Modular response analysis of cellular regulatory networks. $J$ Theor Biol 218, 507-520.

10 Heinrich R \& Schuster S (1996) The Regulation of Cellular Systems. Chapman \& Hall.

11 Klipp E, Herwig R, Kowald A, Wierling C \& Lehrach $\mathrm{H}$ (2005) Systems Biology in Practice. Concepts, Implementation, and Application. Wiley-VCH.

12 Krüger, R \& Heinrich R (2004) Model reduction and analysis of robustness for the Wnt/-Catenin signal transduction pathway. Genome Informatics 15 (1), 138-148.

13 Zobeley J, Lebiedz D, Kammerer J, Ishmurzin A \& Kummer U (2005) A new time-dependent complexity reduction method for biochemical systems. In Transactions on Computational Systems Biology I: Subseries of Lecture Notes in Computer Science (Priomi C ed.) Vol. 3380, pp. 90-110. Springer-Verlag GmbH, Berlin. DOI: $10.1007 / \mathrm{b} 107357$.

14 Hofmeyer JS (2001) Metabolic control analysis in a nutshell. In ICSB 2001. Online Proceedings, http:// www.icsb2001.org/toc.html.

15 Reder C (1988) Metabolic control theory: a structural approach. J Theor Biol 135, 175-201.

16 Kanehisa, M. Goto, S, et al. (2002) The KEGG databases at genomenet. Nucleic Acids Res 30, $42-46$.
17 Schuster S, Kahn D \& Westerhoff HV (1993) Modular analysis of the control of complex metabolic pathways. Biophysical Chem 48, 117.

18 Varga A (2001) Model reduction software in the SLICOT library. In Applied and Computational Control, Signals, and Circuits (629 Kluwer International Series in Engineering and Computer Science) (Datta BN, ed.), pp. 239-282. Kluwer. Academic Publishers, Boston, MA.

19 Benner P, Quintana-Ort ES, Quintana-Ort G (2003) Statespace truncation methods for parallel model reduction of large-scale systems. Parallel Computing 29, 1701-1722.

20 Benner P, Mehrmann V \& Sorensen DC (2005) Dimension Reduction of Large-Scale Systems. Lecture Notes in Computational Science and Engineering. Springer-Verlag, Berlin/Heidelberg.

21 Baur U \& Benner P (2004) Factorized solution of the Lyapunov equation by using the hierarchical matrix arithmetic. Proc Appl Math Mech 4, 658-659.

22 Benner P, Quintana-Ort ES, Quintana-Ort G (2004) Computing passive reduced-order models for circuit simulation. In Proceedings of the International Conference on Parallel Computation in Electric Engineering, PARELEC. pp. 146-151. IEEE Computer Society, Los Alamitos, CA.

23 Saad Y (2003) Iterative Methods for Sparse Linear Systems, 2nd edn. Society for Industrial and Applied Mathematics, Philadelphia, PA.

\section{Appendix}

\section{A derivation of the response coefficients (Eqns 18-20)}

We rewrite Eqn 15 as

$$
\begin{aligned}
\dot{\mathbf{x}}= & A \mathbf{x}+B\left(P \mathbf{s}-\overline{\mathbf{s}}_{\text {bnd }}\right)+B_{\mathrm{p}} \Delta \mathbf{p} \\
\dot{\mathbf{s}}= & N \mathbf{v}(\mathbf{s}, \mathbf{p})+N_{\text {bnd }}\left(\overline{\mathbf{v}}_{\text {bnd }}+C \mathbf{x}+D\left(P \mathbf{s}-\overline{\mathbf{s}}_{\text {bnd }}\right)\right. \\
& \left.+D_{\mathrm{p}} \Delta \mathbf{p}\right)
\end{aligned}
$$

In steady state, the time derivatives vanish, so we set the left hand sides to zero

$$
\begin{aligned}
0= & A \mathbf{x}_{\mathrm{SS}}+B\left(P \mathbf{s}_{\mathrm{ss}}-\overline{\mathbf{s}}_{\text {bnd }}\right)+B_{p} \Delta \mathbf{p} \\
0= & N \mathbf{v}\left(\mathbf{s}_{\mathrm{ss}}, \mathbf{p}\right)+N_{\text {bnd }}\left(\overline{\mathbf{v}}_{\text {bnd }}+C \mathbf{x}_{\mathrm{ss}}+D\left(\mathrm{Ps}_{\mathrm{ss}}-\overline{\mathbf{s}}_{\text {bnd }}\right)\right. \\
& \left.+D_{\mathrm{p}} \Delta \mathbf{p}\right)
\end{aligned}
$$

Now we differentiate the equations by $\Delta \mathbf{p}$ and obtain

$$
\begin{aligned}
0= & A R^{\mathrm{X}}+B P R^{\mathrm{S}}+B_{\mathrm{p}} \\
0= & N\left(\varepsilon R^{\mathrm{S}}+\varepsilon_{\mathrm{p}}\right)+N_{\mathrm{bnd}}\left(C R^{\mathrm{X}}+D P R^{\mathrm{S}}+D_{\mathrm{p}}\right) \\
= & {\left[N \varepsilon+N_{\text {bnd }}\left(D-C A^{-1} B\right) P\right] R^{\mathrm{S}} } \\
& +\left[N \varepsilon_{\mathrm{p}}+N_{\text {bnd }}\left(D_{\mathrm{p}}-C A^{-1} B_{\mathrm{p}}\right)\right]
\end{aligned}
$$

As $A$ is invertible by assumption, Eqn (27) yields Eqn (19). Inserting Eqn (19), Eqn (28) and solving for $R^{\mathrm{s}}$ yields Eqn (18). Eqn (20) follows from differentiation of $\mathbf{v}_{\mathrm{ss}}$, using the chain rule. 